PROCEEDINGS OF THE

AMERICAN MATHEMATICAL SOCIETY

Volume 132, Number 6, Pages 1775-1778

S 0002-9939(03)07297-6

Article electronically published on October 15, 2003

\title{
EXPONENTIAL NONNEGATIVITY
}

\author{
HERBERT WEIGEL
}

(Communicated by Joseph A. Ball)

\begin{abstract}
Let $A$ be a Banach algebra, $a \in A, \sigma(a)$ the spectrum of $a$ and $\tau(a)$ the spectral abscissa of $a$. If $\tau(a) \in \sigma(a)$, then we show that there exists an algebra cone $C \subseteq A$ such that $a$ is exponentially nonnegative with respect to $C$ and the spectral radius is increasing on $C$.
\end{abstract}

Let $A$ be a complex Banach algebra with unit $e,\|e\|=1$. A closed convex and positively homogeneous set $C \subseteq A$ is called an algebra cone if $C \cdot C \subseteq C$ and $e \in C$. If, in addition, $C \cap(-C)=\{0\}$ is valid, then the cone $C$ is called proper. The order induced in $A$ by $C$ is denoted by $\leq$. We denote the spectrum of $a \in A$ by $\sigma(a)$ or occasionally by $\sigma(a ; A)$, the spectral radius of $a$ by $r(a)$ and the spectral abscissa of $a$ by $\tau(a):=\max \{\operatorname{Re} \lambda: \lambda \in \sigma(a)\}$. If $a \in Q(C):=\left\{u \in A: e^{t u} \geq 0, t \in \mathbb{R}^{+}\right\}$, then $a$ is called exponentially nonnegative or quasipositive.

In [5], Theorem 2, Herzog and Schmoeger proved the converse of a Banach algebra version of the Perron-Frobenius Theorem: Let $a \in A, r(a) \in \sigma(a)$. Then there exists a cone $C \subseteq A$ with the property: $a \in C$ and the spectral radius is increasing on $C$. In the matrix case, the converse of the Perron-Frobenius Theorem was already treated in 1968 by Vandergraft (cf. [8] and [2], p. 8).

In the present paper we extend the result of Herzog and Schmoeger to exponentially nonnegative elements of a Banach algebra $A$. The matrix version of this extension was proved in 1970 by Elsner (cf. [3] and [1], p. 80).

I thank the reviewer for his suggestion to treat Example 3.

Theorem. Let $a \in A$. Then the following conditions $\alpha$ ) and $\beta$ ) are equivalent.

$\alpha)$ There exists an algebra cone $C \subseteq A$ such that $a \in Q(C)$ and the spectral radius is increasing on $C: 0 \leq u \leq v \Rightarrow r(u) \leq r(v)$.

B) $\tau(a) \in \sigma(a)$.

Proof. " $\alpha) \Rightarrow \beta$ )" (cf. [4, Theorem 1, (12)): Since $Q(C), \sigma(a)$ and $\tau(a)$ are positively homogeneous, it is no restriction to assume $\sigma(a) \subseteq K:=\{z \in \mathbb{C}:|z| \leq$ $1\}$. Then $\tau(a) \in K$ follows. Because $a \in Q(C)$ and according to the definition of $Q(C)$ we have $\exp (a) \geq 0$ and therefore $r(\exp (a)) \in \sigma(\exp (a))=\exp (\sigma(a))$ is valid (cf. [6], Theorem 5.2). From this we conclude that $\exp (\tau(a))=r(\exp (a)) \in$ $\exp (\sigma(a))$ is true. Since the exponential function is injective on $K, \tau(a) \in \sigma(a)$ follows.

" $\beta) \Rightarrow \alpha) "$ : Let $a \neq 0$ and $b:=\frac{1}{\|a\|} a$. From $\tau(a) \in \sigma(a)$, hence $\tau(b) \in \sigma(b)$, it follows that $\exp (\tau(b)) \in \exp (\sigma(b))=\sigma(\exp (b))$ and thus $r(\exp (b))=\exp (\tau(b)) \in$

Received by the editors October 25, 2002 and, in revised form, February 14, 2003.

2000 Mathematics Subject Classification. Primary 45H05. 
$\sigma(\exp (b))$ are valid. Then according to [5], Theorem 2 there exists an algebra cone $C \subseteq A$ possessing the properties:

1) $\exp (b) \in C$;

2) the spectral radius is increasing on $C$.

It remains to show that $b \in Q(C)$ or equivalently $\exp (t b) \in C$ if $t \in \mathbb{R}^{+}$. Then, since $Q(C)$ is positively homogeneous, $a=\|a\| \cdot b \in Q(C)$ is proved.

For this aim we first describe the cone $C$ (cf. [5], Theorem 2). Let $B:=\Gamma(\Gamma(a)$ ) be the second commutant of $a \in A$, which is a closed commutative subalgebra of $A$. Additionally, the property $\sigma(u ; A)=\sigma(u ; B), u \in B$ is satisfied. Of course $\Gamma(b) \subseteq$ $\Gamma\left(e^{b}\right)$, where $b:=\frac{1}{\|a\|} a$. Since $\Gamma\left(e^{b}\right) \subseteq \Gamma(b)$ (cf. 7], proof of the Theorem), $\Gamma\left(e^{b}\right)=$ $\Gamma(b)$ and therefore $\Gamma\left(\Gamma\left(e^{b}\right)\right)=\Gamma(\Gamma(b))=\Gamma(\Gamma(a))=B$ follows. Moreover, let $\varphi \neq 0$ be a multiplicative linear functional on $B$ satisfying $\varphi(\exp (b))=r(\exp (b))$. Such a $\varphi$ exists, since we showed before that $r(\exp (b)) \in \sigma(\exp (b) ; A)=\sigma(\exp (b) ; B)$. Now the cone $C$ is defined by

$$
C:=\{u \in B: \varphi(u)=r(u)\} \subseteq B .
$$

According to $\varphi(\exp (b))=r(\exp (b))$ we have $\exp (b) \in C$. Now we show that $\exp (t b) \in C$ if $t \in \mathbb{R}^{+}$. Since the multiplicative functional $\varphi$ is continuous, it follows by an easy computation that $\varphi(\exp (t b))=\exp (t \varphi(b))$. From $\exp (\varphi(b))=$ $\varphi(\exp (b))=r(\exp (b)) \in \mathbb{R}$ we conclude that there exists a $k \in \mathbb{Z}$ such that $\varphi(b)=$ $\operatorname{Re} \varphi(b)+2 k \pi i$. Since $\|\varphi\|=1$, we get $2|k| \cdot \pi \leq|\varphi(b)| \leq\|\varphi\| \cdot\|b\|=1$. Thus $k=0$ and therefore $\varphi(b) \in \mathbb{R}$ follows. If $t \in \mathbb{R}^{+}$, we now have $\varphi(\exp (t b))=\exp (t \varphi(b))=$ $(\exp (\varphi(b)))^{t}=(\varphi(\exp (b)))^{t}=(r(\exp (b)))^{t}=(\exp (\tau(b)))^{t}=\exp (t \tau(b))=$ $r(\exp (t b))$. According to the definition of the cone $C$ we finally have $\exp (t b) \in C$ if $t \in \mathbb{R}^{+}$.

Up to now we treated the case $a \neq 0$. Now let $a=0$. Then condition $\beta$ ) is true: $\tau(a)=0 \in\{0\}=\sigma(a)$ and the cone $C:=\left\{t e: t \in \mathbb{R}^{+}\right\}$satisfies condition $\left.\alpha\right)$.

In the course of the proof of the foregoing theorem a commutative algebra $B$ and a cone $C \subseteq B$ were defined. In [5], Theorem 3 it was proved that

$$
\operatorname{rad}(B)=C \cap(-C),
$$

where $\operatorname{rad}(B)$ is the radical of the commutative algebra $B$. Hence if $B$ is semisimple, $\operatorname{rad}(B)=\{0\}$. Then the cone $C$ is a proper cone. The following examples 1 and 2 illustrate this situation.

\section{Example 1.}

Lemma. Let $a \in \mathbb{C}^{d \times d}$. Then $\left.\alpha\right)$ and $\beta$ ) are equivalent. $\alpha$ ) The eigenvalues of a are simple. $\beta$ ) $a \cdot n=n \cdot a, n$ nilpotent $\Rightarrow n=0$.

We only sketch the steps of the elementary proof.

" $\alpha) \Rightarrow \beta$ )": From $\lambda \in \sigma(a): a x=\lambda x, x \neq 0$ we get $a \cdot n x=n \cdot a x=\lambda n x$ and since $\lambda$ is simple $n x=\varrho x, \varrho \in \mathbb{R}$ follows. Now $n$ being nilpotent, the equality $0=n^{l} x=\varrho^{l} x$ for a suitable $l \in \mathbb{N}$ and therefore $\varrho=0$ result. Hence $n x=0$ and since $\mathbb{C}^{d}$ is the linear hull of eigenvectors corresponding to the $d$ different eigenvalues we conclude that $n=0$.

" $\beta) \Rightarrow \alpha$ ": Assume $a$ not to satisfy $\alpha$ ). If 


$$
J:=\left(\begin{array}{ccccc}
\lambda & \delta & & & \\
& \lambda & \delta & 0 & \\
& & \ddots & \ddots & \\
& & & \ddots & \delta \\
& 0 & & & \lambda
\end{array}\right), \delta=0 \text { or } \delta=1
$$

is a $k \times k$ Jordan block $(k>1)$ corresponding to $\lambda \in \sigma(a)$, then the $k \times k$ matrix

$$
n_{k}:=\left(\begin{array}{cccc}
0 & \cdots & 0 & 1 \\
& & & 0 \\
& 0 & & \vdots \\
& & & 0
\end{array}\right)
$$

is nilpotent and commutes with $J$. Using this observation it is easy to construct a nilpotent $n \in \mathbb{C}^{d \times d}, n \neq 0$ that commutes with $a$, a contradiction to assumption $\beta)$.

Corollary. If the eigenvalues of $a \in \mathbb{C}^{d \times d}$ are simple, then the commutative algebra $B:=\Gamma(\Gamma(a)) \subseteq \mathbb{C}^{d \times d}$ is semisimple and the cone $C \subseteq B$ is proper.

Example 2. Let $a=\left(\begin{array}{ll}\alpha & \beta \\ \gamma & \delta\end{array}\right) \in \mathbb{C}^{2 \times 2}$ satisfy $\beta \cdot \gamma \neq 0$. Using the abbreviations

$$
e=\left(\begin{array}{ll}
1 & 0 \\
0 & 1
\end{array}\right), b=\left(\begin{array}{cc}
0 & \beta \\
\gamma & \delta-\alpha
\end{array}\right) \text { and } D=(\alpha-\delta)^{2}+4 \beta \gamma
$$

the following is true: $B:=\Gamma(\Gamma(a))$ is a two-dimensional subalgebra of $\mathbb{C}^{2 \times 2}$ :

$$
B=\{x e+y b: x, y \in \mathbb{C}\}
$$

and

$$
\begin{aligned}
D \neq 0 \Rightarrow & (\sigma(x e+y b)=\{0\} \Leftrightarrow x=y=0) \\
\Rightarrow \quad & C \cap(-C)=\operatorname{rad}(B)=\{0\}, \\
& \text { hence the cone } C \text { is proper; } \\
D=0 \Rightarrow & \left(\sigma(x e+y b)=\{0\} \Leftrightarrow y=\frac{2}{\alpha-\delta} x\right) \\
\Rightarrow & C \cap(-C)=\operatorname{rad}(B)=\left\{x\left(e+\frac{2}{\alpha-\delta} b\right): x \in \mathbb{C}\right\} \neq\{0\}, \\
& \text { hence the cone } C \text { is not proper. }
\end{aligned}
$$

Example 3. Let $A:=\mathbb{C}^{3 \times 3}$ and

$$
a=\left(\begin{array}{rrr}
0 & 1 & 0 \\
-1 & 0 & 0 \\
0 & 0 & 0
\end{array}\right)
$$

Then $\sigma(a)=\{-i, 0, i\}$. According to Theorem 5.2 in [6] there is no algebra cone in $A$ on which the spectral radius is increasing and which contains $a$. Because of $\tau(a)=0 \in \sigma(a)$ it follows from the theorem treated in the present paper, that there is an algebra cone $C \subseteq A$ such that $a \in Q(C)$ and the spectral radius is increasing on $C$. Moreover, by the corollary of the lemma in Example 1 of the present paper, $C$ is a proper cone. 
Using the following notation,

$$
e_{1}:=\left(\begin{array}{ccc}
1 & 0 & 0 \\
0 & 1 & 0 \\
0 & 0 & 0
\end{array}\right) \quad \text { and } \quad e_{2}:=\left(\begin{array}{ccc}
0 & 0 & 0 \\
0 & 0 & 0 \\
0 & 0 & 1
\end{array}\right) \text {, }
$$

we find that

a) $B=\Gamma(\Gamma(a))$ is a three-dimensional subalgebra of $A=\mathbb{C}^{3 \times 3}$ :

$$
B=\left\{x e_{1}+y e_{2}+x a: x, y, z \in \mathbb{C}\right\} \subseteq A
$$

and

$$
e^{t a}=\cos t \cdot e_{1}+e_{2}+\sin t \cdot a \in B, t \in \mathbb{R}^{+}
$$

$\beta$ ) the multiplicative functional $\varphi$ on $B$ defined by

$$
\varphi\left(e_{1}\right)=0, \varphi\left(e_{2}\right)=1, \varphi(a)=0
$$

has the property

$$
\varphi\left(e^{a}\right)=e^{\varphi(a)}=1=r\left(e^{a}\right)
$$

(the last equality follows from $\sigma\left(e^{a}\right)=e^{\sigma(a)}$ );

$\gamma$ ) the cone $C=\{u \in B: \varphi(u)=r(u)\} \subseteq B$ is described explicitly by

$$
C=\left\{u=x e_{1}+y e_{2}+z a \in B: x, y, z \in \mathbb{C} \text { and } y \geq|x \pm i z|\right\} .
$$

Since $e^{t a}=\cos t \cdot e_{1}+e_{2}+\sin t \cdot a, t \in \mathbb{R}^{+}$, it follows that $1=y \geq$ $|\cos t \pm i \cdot \sin t|=\left|e^{ \pm i t}\right|=1$, and therefore $e^{t a} \in C, t \in \mathbb{R}^{+}$.

\section{REFERENCES}

[1] A. Berman, M. Neumann and R. J. Stern, Nonnegative matrices in dynamic systems, Pure and Applied Mathematics, John Wiley and Sons, New York, 1989. MR 90j:93030

[2] A. Berman and R. J. Plemmons, Nonnegative matrices in the mathematical sciences, Academic Press, 1979. MR 82b:15013

[3] L. Elsner, Monotonie und Randspektrum bei vollstetigen Operatoren, Arch. Rational Mech. Anal. 36 (1970), 356-365. MR 40:4804

[4] G. Herzog and R. Lemmert, On quasipositive elements in ordered Banach algebras, Studia Math. 129 (1998), 59-65. MR 99g:46061

[5] G. Herzog and Ch. Schmoeger, A note on a Theorem of Raubenheimer and Rode, Proc. Amer. Math. Soc. 131 (2003), 3507-3509.

[6] H. Raubenheimer and S. Rode, Cones in Banach algebras, Indag. Math. 7 (1996), 489-502. MR 99i:46035

[7] C. Schmoeger, Remarks on commuting exponentials in Banach algebras, Proc. Amer. Math. Soc. 127 (1999), 1337-1338. MR 99h:46090

[8] J. S. Vandergraft, Spectral properties of matrices which have invariant cones, SIAM J. Appl. Math. 16 (1968), 1208-1222. MR 39:5599

Fakultät für Mathematik, Universität Karlsruhe, D-76128 Karlsruhe, Germany

E-mail address: herbert.weigel@math.uni-karlsruhe.de 